CUAD. CONTAB. / BOGOTÁ, COLOMBIA, 16 (40): 179-204 / ENERO-ABRIL / 179

\title{
Bases para un modelo de estructura de financiamiento en las pyme latinoamericanas*
}

doi:10.11144/Javeriana.cc16-40.bpme

\section{Jorge Orlando Pérez}

Doctor en contabilidad, Universidad Nacional de Rosario, UNR. Magíster en dirección de empresas, Universidad Católica de Córdoba, UCC. Contador público, Universidad Católica de Córdoba, UCC. Profesor titular e investigador, Universidad Católica de Córdoba, unidad asociada al Consejo Nacional de Investigaciones Científicas y Técnicas, CONICET y Universidad Nacional de Villa María, UNVM. Correo electrónico: jorgeoperez53@gmail.com

\section{Martín Bertoni}

Contador público, Universidad Católica de Córdoba, UCC. Jefe de trabajos prácticos e integrante de equipo de investigación en la Universidad Católica de Córdoba, unidad asociada al Consejo Nacional de Investigaciones Científicas y Técnicas, CONICET.

Correo electrónico: bertonimartin@gmail.com

\begin{abstract}
Silvana Andrea Sattler
Licenciada en economía, Universidad Nacional de Córdoba, UNC. Profesor ayudante e integrante de equipo de investigación en la Universidad Nacional de Córdoba e integrante de equipo de investigación en la Universidad Católica de Córdoba, unidad asociada al Consejo Nacional de Investigaciones Científicas y Técnicas, CONICET. Correo electrónico: silsattler@gmail.com
\end{abstract}

\section{Dante Domingo Terreno}

Contador público, Universidad Nacional de Córdoba, UNC. Profesor adjunto e integrante de equipo de investigación e integrante de equipo de investigación en la Universidad Católica de Córdoba, unidad asociada al Consejo Nacional de Investigaciones Científicas y Técnicas, CONICET y de la Universidad Nacional de Córdoba. Correo electrónico: danteterreno@fibertel.com.ar

\footnotetext{
* El trabajo se deriva del proyecto bienal (2014-2015) de investigación radicado en la Universidad Católica de Córdoba, con el título Un modelo para establecer la estructura óptima de financiación de las pymes argentinas, director Jorge Orlando Pérez, cuyo objeto es diseñar un modelo que permita a la pyme argentina establecer su estructura de financiamiento adecuada, en relación con el riesgo que pueda asumir y sus expectativas de rentabilidad.
} 
Resumen Este trabajo se propone resolver el problema de la determinación de la estructura de financiamiento para cada pyme. En la mayoría de las investigaciones sobre este tema, se realizan análisis descriptivos acerca de las variables que inciden en la decisión sobre la mezcla entre pasivo y patrimonio en contextos diferentes, mientras que en este caso se aporta una herramienta, que se espera mejore el proceso de la toma de decisiones financieras. Para abordar el tema planteado, se propone un modelo matemático para que el administrador financiero de la pyme pueda establecer la mezcla de capitales más conveniente. Para ello, previamente, se identifican las características de las distintas fuentes de financiamiento, las variables que inciden en el riesgo y la asociación entre la fuente y el destino de los fondos. Se desarrolla la fundamentación matemática del modelo y luego se traslada el mismo a una planilla de cálculo electrónica, para que, al cargar los datos de las distintas variables, se pueda arribar a la mejor combinación entre fondos propios y ajenos, de manera que al confrontar con la rentabilidad de la inversión, se pueda obtener el mayor valor económico agregado.

Palabras clave pequeñas y medianas empresas; capital; estructura financiera; riesgo de insolvencia.

\section{Código JEL M400}

\section{Basis for a Funding Structure Model for Latin American Pymes}

\footnotetext{
Abstract This work aims to solve the problem of determining the funding structure for each pyme (small and mid-sized companies). Most of the research on this topic shows the carrying out of a descriptive analysis on the variables that influence the decision of mixing liabilities and equity in different contexts, while in this case we provide a tool that it is expected to improve the financial decision-making process. To introduce the established topic we propose a mathematical model for the financial manager of the pyme can establish the most convenient mix of capitals. For this, and previously, we identify the charac-
}

teristics of the different sources of funding, the variables that influence the risk, and the association between the source and the destination of the funds. We develop the mathematical foundation of the model and then it is taken to an electronic calculus form so, upon providing the data for the different variables, the best combination between own funds and borrowed funds can be achieved, so when contrasting these with the profitability of the investment a higher economic value added can be obtained.

Keywords Small and mid-sized companies; capital; financial structure; insolvency risk.

\section{Bases para modelo de estrutura de financiamento nas PME latino- americanas}

Resumo Este trabalho visa resolver o problema da determinação da estrutura de financiamento para cada PME. Na maioria das pesquisas sobre este tema são realizadas análises descritivas acerca das variáveis que incidem na decisão sobre a combinação entre passivo e patrimônio em contextos diferentes, contudo, neste caso, fornece-se uma ferramenta que, achamos, vai melhorar o processo da tomada de decisões financeiras. Para abordar o tema colocado, propõe-se um modelo matemático para o administrador financeiro da PME puder estabelecer a combinação de capitais mais conveniente. Para isso, previamente são identificadas as características das diferentes fontes de financiamento, as variáveis que incidem no risco e a associação entre fonte e destino dos fundos. Desenvolve-se a fundamentação matemática do modelo que é trasladado, em seguida para uma folha de cálculo eletrônica, visando que, ao carregar os dados das diferentes variáveis, seja possível acessar à melhor combinação entre fundos próprios e alheios, de maneira que no confronto com o retorno do investimento, poderá se obter o maior valor econômico agregado.

Palavras-chave Pequenas e médias empresas; capital; estrutura financeira; risco de insolvência. 


\section{Introducción}

Establecer las fuentes de financiamiento que permitan optimizar el capital, constituye un problema significativo en cualquier empresa; esta situación se complica cuando se trata de una pyme, por las restricciones que debe enfrentar, más aún en contextos inestables como el de algunos países latinoamericanos. Por ello, en este trabajo se brindará un instrumento que coadyuve en cada caso a obtener la mezcla óptima de financiamiento.

La cuestión referida a la estructura de capital ha merecido un importante tratamiento en las finanzas; se han postulado diversas teorías que han ido profundizando el tema, cuyos primeros aportes fueron analizados en condiciones de mercados desarrollados. La teoría tradicional tuvo su impulso a mediados del siglo XX; David Durand (1952) es uno de sus principales propulsores y postula que un moderado uso del endeudamiento permite reducir el costo del capital, al generar el crecimiento del valor de la empresa. Si el uso del capital ajeno se incrementa, crece el riesgo de insolvencia y este se manifiesta en la caída del valor de las acciones. La empresa debe acertar en la mezcla de financiamiento, que se alcanza cuando el costo del capital se hace mínimo y el valor de la empresa se hace máximo (López-Dumrauf, 2003).

Posteriormente, Franco Modigliani y Merton H. Miller (1958) irrumpieron con su teoría, al sostener que el costo de capital promedio ponderado es constante e independiente de las proporciones entre capitales propios y ajenos. Al incrementarse el endeudamiento, aumenta el riesgo de cesación de pagos, pero la mayor tasa de interés que exigirían los acreedores se compensa con el menor rendimiento que requerirían los accionistas. Según esta tesis, el administrador financiero debe enfocarse en el rendimiento de sus activos, ya que la decisión de financiamiento pasa a ser irrelevante. Estos postulados serían válidos en un mercado eficiente, en el cual las anomalías de precios y tasas se anulen rápidamente.

En una segunda proposición, Franco Modigliani y Merton H. Miller (1959) sostienen que la rentabilidad de la firma es una función lineal y directa de su endeudamiento multiplicada por el diferencial de rendimiento, sumando la tasa básica de costo de capital, todo a valores de mercado; mencionan una proporción de endeudamiento máximo para cada sector de la economía.

En un artículo posterior, Modigliani y Miller (1963) generan una tercera proposición, en la que desarrollan el efecto impositivo en la toma de financiamiento de los acreedores. $\mathrm{Al}$ ser deducibles los intereses del impuesto a las ganancias, parte del costo del endeudamiento es soportado por el gobierno y el valor de la empresa se ve incrementado por el efecto fiscal (Drimer, 2011). Más tarde, Merton H. Miller (1977) introduce el efecto impositivo sobre la renta personal.

A partir de entonces, distintos autores comenzaron a desarrollar aspectos relacionados con las imperfecciones de los mercados y surgieron cuestiones como los costos de agencia (Diamond, 1989; Fama \& Miller, 1972; Jensen, 1986; Jensen \& Meckling, 1976), la información asimétrica (Petersen \& Rajan, 1994; Stiglitz 
\& Weiss, 1981), la teoría del equilibrio estático o trade-off theory (Brennan \& Schwartz, 1978; Kim, 1978; Kraus \& Litzenberger, 1973) y las jerarquías financieras (Myers, 1984; Myers \& Majluf, 1984). A continuación, se describe el concepto fundamental que estas teorías consideran.

Costos de agencia. Analiza los conflictos de intereses entre los propietarios del capital y sus administradores, y propone que es posible buscar una estructura de financiamiento óptima que minimice los costos de agencia y maximice el valor de la empresa. El supuesto esencial de esta teoría es que el rol del inversionista y el administrador no se configuran en una misma persona.

Teoría de la información asimétrica. Los distintos agentes no tienen acceso al mismo nivel de información, lo que genera decisiones antieconómicas por el hecho de no recibir información adecuada, lo cual incide al momento de buscar financiamiento.

Teoría del trade-off o del equilibrio de la estructura de capital. A partir de los costos en que se incurriría al tener dificultades financieras y de los costos de agencia, se busca establecer la combinación óptima de las fuentes financieras.

Teoría de la jerarquía de preferencias (pecking order theory). Afirma que no hay una estructura óptima de financiamiento, sino que los administradores recurren a una escala de jerarquías, al utilizar en primer lugar los fondos autogenerados, con base en la asimetría de la información que les dificulta el acceso al financiamiento externo.

Más recientemente, a finales del siglo XX, surgieron las finanzas conductuales (LeRoy,
2004; Schweitzer, 2008; Stein, 1997), que cuestionan el enfoque racional de las decisiones financieras.

Los aportes mencionados anteriormente contribuyeron al estudio del financiamiento en general. No obstante, la aplicación de estas teorías en pequeñas y medianas empresas que operan en mercados poco desarrollados, no refleja la realidad de estas y por ende, podría conducir a conclusiones equivocadas. Si bien se han efectuado diversos aportes para el caso particular de estas entidades, este trabajo pretende ampliar el enfoque hacia las pyme que realizan sus actividades en mercados imperfectos.

En un trabajo desarrollado por Ricardo Bebczuk (2010), al referirse a las restricciones de financiamiento que tienen las distintas empresas, sostiene:

\begin{abstract}
No hay diferencias tajantes en la restricción percibida por empresas grandes y pequeñas, aunque sí entre países con distinto nivel de desarrollo financiero. Este hecho revela que los factores macroeconómicos tienen una incidencia más significativa que las diferencias en el grado de asimetría informativa entre empresas de distinto tamaño.
\end{abstract}

Sin embargo, algunos autores - como Sheridan Titman y Roberto Wessels (1988)— afirman que las empresas pequeñas presentan mayor probabilidad de quiebra y más dificultades para acceder a los mercados de capitales.

Hay aspectos característicos de las pyme que condicionan el proceso decisorio. Los problemas derivados de la unificación entre propiedad y dirección, la asimetría de la información 
brindada a terceros, la influencia de las decisiones familiares y la incidencia de los aspectos emocionales son los más comunes y significativos. A continuación, comentaremos brevemente sobre cada uno de ellos:

La unificación entre dirección y propiedad. En contraposición al problema que se trata en la teoría de la agencia, por lo general en este tipo de entes se confunden las figuras del aportante del capital y la administración. Anahí Briozzo y Hernán Vigier (2009) señalan que este hecho genera subjetividades al momento de la toma de decisiones, ya que estas firmas no están expuestas a la evaluación del mercado de capitales.

Asimetría en la información que brindan a terceros. Joseph Stiglitz y Andrew Weiss (1981) sostienen que este aspecto da lugar al racionamiento de la oferta de crédito. También en un estudio empírico, José Pablo Dapena y Juan Lucas Dapena (2003) demuestran que la falta de transparencia en la información que presentan las pyme conlleva dificultades en la consecución de fondos del sistema financiero.

Influencia de las decisiones familiares. Al estar compuestas las pyme en su mayoría por integrantes del grupo familiar, el proyecto de estos suele estar unido al de la empresa, por lo que las consideraciones sobre el riesgo y la pérdida de control adquieren connotaciones diferentes, marcadas fuertemente por los intereses personales. Peter Leach (2010, p. 39) señala:

En el ámbito familiar, que se fundamenta en aspectos emocionales, prevalecen la protección y la lealtad, mientras que en la esfera empresarial, sustentada en la tarea, rigen el rendimiento y los resultados. La empresa fa- miliar es una fusión de estas dos poderosas instituciones y si bien proporciona el potencial para lograr un rendimiento superior, no es raro que eso también lleve a enfrentar serias dificultades. Esto puede significar que los patrones de conducta - emocionales-que surgen dentro de la empresa, en un contexto comercial, son profundamente irracionales e inapropiados.

Significativo impacto de las emociones. Las neurociencias han comprobado que las decisiones no siempre son racionales. George A. Akerlof y Robert J. Shiller (2009, p. 17) manifiestan:

Para comprender el funcionamiento de las economías y cómo podemos gestionarlas para prosperar, debemos prestar atención a los patrones de funcionamiento que influyen en las ideas y los sentimientos de las personas, es decir, sus espíritus animales. Solamente podremos llegar a comprender con certeza los acontecimientos económicos relevantes si sabemos enfrentarnos con el hecho de que sus causas son principalmente de carácter mental.

En las pyme la incidencia de las emociones en la toma de decisiones puede incluso ser superior a la de organizaciones más grandes, por la influencia de los aspectos que se señalan en la unificación entre dirección y propiedad y el impacto de las decisiones familiares.

Si bien las cuestiones fundamentales que tienen relación con la maximización del valor de la firma se refieren a decisiones de inversión 
y de financiación, por lo general, las primeras se consideran como las más importantes, ya que un error en la toma de decisiones sobre el primer aspecto, puede llevar a resultados negativos irreversibles, mientras que la búsqueda de fondos podría ser una cuestión menos complicada. Sin embargo, la realidad no siempre opera de esta manera, tal como afirma Ricardo Pascale (2009, p. 259):

Es frecuente encontrar en la literatura especializada la afirmación de que es mucho más difícil hacer ingresar a una empresa con serios problemas de rentabilidad y estabilidad financiera con base en decisiones de financiamiento que a partir de las decisiones de inversión.

En muchos casos, la experiencia de países de América Latina fue otra. Ciertas decisiones de financiamiento han hecho inviables los procesos de recuperación de las empresas. Basta recordar el componente en moneda extranjera en el financiamiento de una empresa, pues esta política se podría seguir si se supone el mantenimiento de una determinada política cambiaria. Cambios abruptos en esta (por ejemplo, una fuerte devaluación) han llevado a muchas empresas a la pérdida de todo su capital y a una situación de insolvencia o de bancarrota.

En las pyme en particular, un número importante de autores - como Anahí Briozzo y Hernán Vigier (2008), Paola Brighi y Giuseppe Torluccio (2007); Denis Forte, Lucas Ayres B. de C. Barros y Wilson Toshiro Nakamura (2013); Ciarán Mac an Bhaird y Brian Lucey
(2011) y Claudio Romano, George A. Tanewski y Kosmas Smyrnios (2000) — ha abordado el estudio de los determinantes de la estructura de financiación en este tipo de empresas, que mostraron evidencias significativas y aportes de valor para su estudio.

Los trabajos mencionados y otros de similar importancia han sido estudios empíricos que analizaron las causas por las cuales las pyme toman determinada decisión acerca del modo en que se financian. Así, detectaron cuestiones como la correlación del contexto macroeconómico, los cambios que se operan a medida que el ciclo de vida de la empresa y de sus propietarios se modifica, el orden de jerarquía de las fuentes de financiamiento o las condiciones internas que condicionan la decisión.

En general, los aportes realizados por distintos autores enfocados en la problemática de las pequeñas y medianas empresas, se centran en un análisis descriptivo de la manera en que estas efectúan la mezcla de capitales que financian la inversión. En este trabajo, el énfasis está puesto en el administrador financiero que debe enfrentarse a la decisión de lograr la mejor combinación entre pasivo y patrimonio.

Obtener un modelo matemático que sirva para determinar la estructura óptima de financiamiento para las pyme es un objetivo que se plantea este trabajo, basado en aspectos ligados a mercados no desarrollados como los latinoamericanos, teniendo en cuenta que no hay una sola distribución de capital aplicable para todas las empresas, tal como señalan Richard A. Brealey, Stewart C. Myers y Franklin Allen (2006, p. 394) en una buena analogía: 
Encontrar el ratio de endeudamiento óptimo es como encontrar el límite de velocidad óptimo, se puede estar de acuerdo en que los accidentes a 30 millas por hora son normalmente menos peligrosos que los accidentes a 60 millas por hora, pero eso no significa que debemos fijar el límite de velocidad en 30 millas por hora para todas las carreteras. La velocidad tiene beneficios, igual que el riesgo, y también los tiene el endeudamiento...

Para lograr el objetivo propuesto, se describirán las principales fuentes de financiamiento para estas empresas en el contexto referido, ya que su elección incidirá en el modelo propuesto. Posteriormente, se analizarán las variables de riesgo tanto operativo como financiero, que influenciarán el costo del capital. Por otra parte, se considera necesario examinar el destino de los fondos, ya que el tipo de financiamiento habitualmente debe estar asociado a las características de la inversión.

Finalmente, se avanza hacia la elaboración de un modelo matemático que contemple las variables señaladas y sirva para determinar en cada caso, la mejor estructura de financiamiento que maximice la brecha entre los costos y la rentabilidad de los activos.

\section{Fuentes de financiamiento}

Al momento de seleccionar las fuentes de financiamiento, es necesario realizar una clasificación más amplia que la sola referencia a pasivo o patrimonio, para atender a las características de cada una, lo que provocará un orden de jerarquía para el financiamiento, según el acceso, el destino, el costo y el riesgo. En este caso, se categorizarán en pasivos operativos, pasivos financieros, aportes de capital y resultados no distribuidos.

Pasivos operativos. Fundamentalmente se generan por medio de los proveedores de bienes y servicios; en menor medida por las deudas fiscales y de seguridad social. También hay empresas que se financian con anticipos de sus clientes.

Pasivos financieros. Los préstamos de entidades financieras y en algunos casos, la emisión de obligaciones negociables.

Aportes de capital. Esta categoría se puede clasificar entre actuales propietarios por un lado $y$, potenciales inversores por otro. Salvo el capital inicial, a posteriori no es una vía que las pyme utilicen con frecuencia, debido a la escasa apertura al mercado de capitales y al temor por la pérdida de control.

Resultados no distribuidos. Los fondos que provienen del autofinanciamiento no constituyen una función lineal. En las pyme, la decisión de distribuir o no resultados no está solo en relación con el nivel de las ganancias y la necesidad de reinversión, sino también con los requerimientos de los propietarios. Para estos, los retiros de fondos suelen ser su único medio de vida y habitualmente, operan en forma anticipada a la generación de resultados; por tanto, no pueden caer a determinados niveles. Cuando hay bajos resultados, la propensión marginal a retenerlos suele ser también baja, a pesar de que la necesidad financiera resulte importante.

La teoría de la jerarquía financiera (pecking order theory), desarrollada por Stewart C. Myers (1984) y Stewart C. Myers y Nicholas S. Majluf 
(1984), sostiene que a raíz de las asimetrías de la información no se conoce el valor presente neto de las inversiones, lo que implica que se emita deuda o capital por debajo de su valor real de mercado, lo que lleva a estas empresas a inclinarse por el financiamiento con recursos propios, fundamentalmente por medio de resultados no distribuidos.

En una investigación desarrollada en Australia, Adrian Zoppa y Richard G. P. McMahon (2002) proponen una jerarquía específica para pyme y llegan a la conclusión de que las fuentes de financiamiento en este tipo de empresas se seleccionan en el siguiente orden: 1) reinversión de beneficios; 2) financiamiento con deuda a corto plazo, incluyendo crédito comercial y préstamos personales; 3) financiamiento con deuda a largo plazo, comenzando con préstamos de propietarios, amigos y familiares; 4) nuevos aportes de capital de propietarios, familiares y amigos; 5) aportes de capital de terceros.

Un estudio realizado por la Fundación de Investigaciones Económicas Latinoamericanas (FIEL, 1996) sobre el financiamiento de las pyme, determina que en Argentina las principales fuentes en orden de importancia son la reinversión de utilidades, el crédito bancario o financiero local, el financiamiento de proveedores, el aporte de socios y el crédito del exterior.

\section{El riesgo}

Una de las decisiones fundamentales que debe observarse al tomar financiamiento radica en que el costo del capital resulte inferior a la rentabilidad de los recursos invertidos. Si los fondos provistos por terceros tienen un costo (neto del efecto impositivo) inferior a los propios, la tendencia será recurrir a los primeros, lo que provoca una elevación del ROE (rentabilidad del patrimonio), por medio del leverage o apalancamiento financiero favorable.

No obstante lo anterior, es importante tomar en cuenta que al aumentar el pasivo, los proveedores de fondos estarán propensos a incrementar la tasa (por el aumento del riesgo) y por otra parte, hay que considerar que el rendimiento de los activos no es lineal (la mayor inversión no implica que la rentabilidad continúe siendo la misma). Teniendo en cuenta esta afirmación, será necesario identificar los factores que inciden sobre los riesgos operativo y financiero.

El riesgo operativo estará dado por la volatilidad y las perspectivas del EBITDA (sigla de Earnings before Interests, Taxes, Depreciations and Amortizations, ganancia antes de intereses, impuestos, depreciaciones y amortizaciones).

Volatilidad del EBITDA. Cuando se observa que la desviación estándar del EBITDA en períodos anteriores presenta valores erráticos, esta situación produce un alejamiento de la certidumbre sobre la consecución de ganancias futuras.

Perspectivas del EBITDA. En este punto, se analizarán fundamentalmente las expectativas de evolución del mercado y la inserción de la firma dentro de este. En este sentido, es importante tener en cuenta la estructura de costos, ya que una elevada incidencia de costos fijos, ante 
una caída en el nivel de operaciones, impactará negativamente los resultados.

El riesgo financiero estará influenciado por la evolución de la estructura de financiamiento, la cobertura de los intereses con el flujo de efectivo operativo, la capacidad de devolución, el plazo, las políticas macroeconómicas, la existencia de deudas en moneda extranjera, el tipo de activo y el tramo de financiamiento.

Evolución de la estructura de financiamiento. Será necesario observar cómo evolucionan la estructura de financiamiento y la relación pasivo/patrimonio, para verificar que no se produzca un desequilibrio que ponga en peligro el respaldo a los acreedores.

Cobertura de los intereses. El flujo de efectivo operativo debe mostrar suficiencia para hacer frente a los intereses. En la medida en que estos últimos asuman una proporción importante respecto a los fondos autogenerados, el riesgo de tener inconvenientes financieros aumenta.

Capacidad de devolución. Para verificar si los servicios de la deuda podrán ser atendidos, se puede establecer un indicador a partir de un desarrollo de Krishna G. Palepu, Paul M. Healy y Victor L. Bernard (2002, pp. 14-18):

$$
c f e=\frac{E B I T D A}{i+\frac{p d}{(1-t i)}+\frac{d p}{(1-t i)}}
$$

Donde:

cfe = cobertura del flujo de efectivo

EBITDA = utilidad antes de intereses e impuesto a las ganancias

$i \quad=$ intereses del pasivo

$$
\begin{array}{ll}
p d & =\text { pago de la deuda } \\
t i & =\text { tasa de impuesto } \\
p d & =\text { dividendos preferentes }
\end{array}
$$

Se excluyen los dividendos comunes, porque son evitables. La división tanto de los pagos de deuda como de los dividendos preferentes por uno menos la tasa de impuesto, obedece a que, al no ser estos pagos deducibles fiscalmente, se les exige un valor mayor que al pago de intereses (ya que estos se descuentan para el cálculo del impuesto). En el caso de las pyme, no siempre distribuyen dividendos preferentes; en su lugar, habría que considerar retiros mínimos de los socios.

Cuando la ratio propuesta $c f e$ (cobertura del flujo de efectivo) arroja un resultado por debajo de la unidad, muestra la generación de un flujo de fondos insuficiente para hacer frente a compromisos ineludibles.

El plazo. Cuando el financiamiento es proporcionalmente alto en el corto plazo, ello incidirá en el aumento del riesgo para la empresa, pues las obligaciones podrían no renovarse a su vencimiento o incurrir en mayores costos de financiación. Este aspecto toma mayor relevancia en contextos cambiantes y de racionamiento del crédito, el cual se entiende que opera en forma inversa a los acreedores, ya que estos habitualmente consideran más riesgosa la operación financiera cuando el plazo es prolongado.

Políticas macroeconómicas. Algunos estudios empíricos como el de José María Fanelli, Ricardo N. Bebczuk y Juan J. Pradelli (2003) han comprobado que cuando la volatilidad del contexto macroeconómico es mayor, esto afecta la 
estructura de capital de las firmas, aunque de manera diferente según su capacidad para acceder al financiamiento.

Deudas en moneda extranjera. Este aspecto toma particular relevancia en los países en desarrollo que suelen verse afectados por crisis en la balanza de pagos, lo que deriva en variaciones del tipo de cambio, que generan - en aquellos casos en que no se cuente con ingresos suficientes desde el exterior-, un desbalance entre los flujos de fondos externos que deriva en trastornos financieros.

El tipo de activo. Richard A. Brealey, Stewart C. Myers y Franklin Allen (2006, p. 531) señalan que en caso de que la quiebra deteriore el valor de los activos, habrá una propensión a tomar menos deuda. Estos autores dan el ejemplo de la industria farmacéutica y de ciertos servicios que tienen un bajo endeudamiento, porque el valor de la empresa depende del capital humano que puede caer más rápido. En este trabajo, se sostiene que este aspecto puede ser trasladado en gran medida a las pyme, pues en muchos casos, el valor de la empresa está atado al conocimiento y al trabajo de sus integrantes.

Tramo de financiamiento. Siguiendo a Roberto Drimer (2011, p. 61), opinión que se comparte en este trabajo, es necesario separar el financiamiento por intervalos, ya que los costos no serán uniformes en todo el tramo de requerimiento de inversión y por tanto, el riesgo cambiará en cada etapa.

A medida que aumenta la proporción de pasivos y superado cierto límite, comienzan a incrementarse riesgos de insolvencia. Zvi Bodie, Robert Merton y Guillermo Vinitzky (2006, p. 524) expresan: "la simple amenaza de insolvencia financiera puede ser costosa para la empresa amenazada. Los clientes y proveedores se muestran reticentes a hacer negocios con una empresa a la que puede no quedarle demasiado tiempo".

Por ello, ante similares niveles de costos tanto en el capital propio y en el ajeno, un elevado endeudamiento puede hacer inclinar la balanza en la decisión hacia el capital propio. Este fenómeno toma mayor dimensión en las pyme, pues la sensibilidad de los terceros puede causar un mayor impacto.

El riesgo de insolvencia es un aspecto a considerar no solo por los proveedores del financiamiento, sino que también debe ser tomado en cuenta internamente, tal como señala Guillermo López-Dumrauf (2003, p. 440):

$$
\begin{aligned}
& \text { Cuando aumenta el endeudamiento, el au- } \\
& \text { mento del riesgo de insolvencia se reparte } \\
& \text { entre accionistas y obligacionistas. Por lo } \\
& \text { tanto, el rendimiento exigido a las acciones } \\
& \text { — ke - se calcula sumando una prima más o } \\
& \text { menos fija al rendimiento exigido a la deu- } \\
& \text { da }-k d-\text {. } \\
& \text { Los costos de insolvencia financiera hacen } \\
& \text { que se modifique el riesgo de los activos } \\
& \text { (medido por } \beta \mu \text { ) y también el rendimiento } \\
& \text { exigido a estos, } k u \text {. }
\end{aligned}
$$

Para las pyme, cuando el pasivo supera cierto nivel, corresponderá estimar el costo por riesgo de insolvencia, el cual se establecerá en función de la aversión al riesgo de los propietarios y crecerá a medida que aumenta el financiamiento de terceros. 


\section{Destino de los fondos}

Tal como se sostiene en un trabajo de Jorge Orlando Pérez (2013), la aplicación de los recursos financieros incide sobre la fuente de financiamiento a considerar.

Constituye una cuestión relevante analizar dónde serán destinados los fondos, ya que un uso ineficiente de los recursos puede derivar en una imposibilidad de pago en el futuro. El nuevo pasivo podrá financiar el activo corriente o las inversiones de largo plazo; según donde vaya dirigido, merecerá distintos análisis.

Al tratarse de un préstamo para fortalecer el capital de trabajo, deberá evaluarse lo siguiente: 1) ¿el fondo de maniobra actual es el necesario?, 2) ¿la velocidad de circulación del efectivo es razonable?, 3) ¿la insuficiencia de capital circulante no se debe a que los recursos de corto plazo son absorbidos por las actividades de inversión o de financiación? y 4) ¿se prevén mayores ventas?

Para saber si el fondo de maniobra actual es suficiente, se debe considerar la composición de los activos y pasivos corrientes y los futuros flujos de efectivo, con la finalidad de determinar la suficiencia del capital de trabajo neto. Si este último es acorde o excede sus necesidades y el nivel de operaciones se mantiene, un incremento del mismo generará fondos ociosos, lo cual - por ende-perjudica la rentabilidad futura.

La velocidad de circulación de los fondos de corto plazo es un aspecto importante para considerar la inversión en capital de trabajo. Por ejemplo, suele ser una fuente de problemas cuando se cuenta con un plazo de cobranzas su- perior al concedido originalmente a los clientes o que los inventarios estén inmovilizados por un tiempo mayor al razonable. Ante los casos mencionados, lo prudente es corregir primero las distorsiones, antes de tomar financiamiento adicional.

Si la insuficiencia de fondos se debe a la absorción del flujo operativo por medio de las actividades de financiación o de inversión, ello posiblemente obedece a un error en la política financiera y habrá que evaluar si estas actividades seguirán demandando recursos en el futuro. En ese caso, el nuevo financiamiento habrá que analizarlo en función de las nuevas inversiones, los servicios de la deuda o la distribución de resultados y no en relación con el capital de trabajo.

De resultar previsible un fuerte incremento de las ventas, siempre que antes de esa situación el capital de trabajo fuese el mínimo necesario, la relación ventas/capital corriente no debe aumentar, es decir, el capital de trabajo debe incrementarse en una proporción similar al nivel de actividad; porque de no ser así, significaría una escasez del fondo de maniobra para abastecer las mayores erogaciones que se generarían por arrastre de las ventas (salvo que el plazo de pago supere al ciclo de operaciones), lo que puede provocar un estrangulamiento financiero de corto plazo, debido a que los recursos no estarán disponibles para cancelar el mayor saldo de proveedores que se origine para financiar el aumento de inventarios.

En caso de que la deuda se destinara a financiar el activo fijo - como este en la gran mayoría de los casos genera un flujo de fondos periódico que tiene una baja proporción en relación con la inversión-, deberá tomarse la precaución de 
que no se atienda con fondos de corto plazo, a no ser que haya un excedente de capital de trabajo y pueda ser volcado a esta finalidad.

Para la situación descrita en el párrafo anterior, por lo general debe preverse que los fondos permanentes (pasivos de largo plazo y recursos propios) sean superiores a la inversión fija, para lo cual se utiliza el siguiente indicador:

\section{$\underline{\text { Pasivo no corriente }+ \text { Patrimonio }}$ Activo no corriente}

En caso de que la ratio anterior resultara inferior a la unidad, significará que parte de los activos no corrientes está siendo financiada con pasivos de corto plazo, lo cual implicaría una señal de alarma, ya que los primeros normalmente no generan suficiente flujo de fondos, para enfrentar las obligaciones contraídas en ese lapso.

\section{Premisas para un modelo}

El modelo para tomar decisiones de financiamiento en las pyme, que aquí se presenta, está basado en los pilares que a continuación se detallan.

Capital propio mínimo. Se debe partir de un nivel de recursos propios suficiente, ya que los acreedores estarán dispuestos a asumir riesgo en forma limitada.

Separación de los pasivos operativos de los financieros. Los pasivos operativos están asociados generalmente al capital de trabajo y presentan costos diferentes a los financieros (en algunos casos, nulos o irrelevantes). Se considera que debe partirse de un pasivo de este tipo mínimo.
Aumento del costo financiero por causas exógenas y endógenas. A medida que se incrementa el pasivo, crece la tasa de interés (por el mayor riesgo al que están expuestos los acreedores) y en su costo financiero, la empresa considera el aumento del riesgo de insolvencia (por la mayor exposición de la entidad a caer en dificultades financieras).

Tramos de inversión. El análisis se debe realizar por intervalos, ya que se espera que en cada uno de ellos varíe el costo de los pasivos (con base en el riesgo considerado por los acreedores e internamente por el costo del riesgo de insolvencia) y la generación de resultados en relación con el activo.

Rentabilidad y costo incremental. La mezcla de las fuentes financieras que tenga el costo de capital más bajo y, a su vez, resulte inferior a la rentabilidad de la inversión, mostrará la conveniencia de la estructura de capital. Es importante comparar el rendimiento del activo incremental frente al costo del financiamiento adicional, ya que un cotejo global puede inducir a tomar decisiones erróneas (es posible que un determinado financiamiento tenga un costo superior al retorno de la inversión y que, sin embargo, el costo promedio arroje una tasa más baja).

\section{Objetivo y supuestos del modelo que se desarrolla en este trabajo}

El objetivo del modelo es determinar la existencia de un punto de indiferencia en la decisión de financiarse con fondos propios o de terceros, ante la alternativa del aumento de la inversión, para validar la existencia de un umbral en la mezcla de 
fondos, a partir del cual ya no conviene a las empresas alterar la mezcla de financiamiento obtenida, dado que la determinada es la que minimiza los costos totales de esta estructura.

A continuación, se desarrollan los supuestos sobre los que se basa el modelo:

Las variables de decisión a analizar son las "fuentes de financiamiento incrementales".

Hay restricciones al acceso al mercado de capitales y racionalización del crédito proveniente del mercado financiero.

No se convalida el presupuesto de mercado eficiente, de modo que la tasa de interés no se ajusta de acuerdo a las fluctuaciones del mercado y de manera común y homogénea para agentes similares, sino que puede variar para cada potencial deudor en relación con sus características endógenas.

Hay una estructura de inversión necesaria para el negocio. Esto se considera un parámetro dado en el modelo; la determinación de su magnitud y composición no forma parte del análisis.

Hay dos posibles fuentes de financiamiento, en razón de su procedencia: de propietarios y de terceros. Los fondos de los propietarios pueden considerarse por aportes y por resultados no distribuidos. Los pasivos comprenden los operativos y los financieros.

Se exige un patrimonio mínimo, para el cual los acreedores estén dispuestos a financiar, en parte, la estructura de inversión del negocio. Este parámetro supone una relación determinada respecto del nivel de inversión dado y necesario al negocio.

Se supone la existencia de un conjunto de pasivos mínimos asociados a las deudas operativas de corto plazo propias de la estructura comercial del negocio. Estas deudas se distinguen de las financieras (en principio las que analizaríamos como incrementales), entre otras cosas, por estar sujetas a tasas de interés diferenciales. Este parámetro supone una relación determinada respecto del nivel de inversión dado y necesario al negocio.

Los costos financieros asociados a los pasivos incrementales son deducibles de la utilidad neta para la determinación del impuesto a las ganancias, no así en las utilidades distribuibles a los propietarios.

El rendimiento del negocio (ROA) se plantea para distintos tramos de inversión. Es decir, no es una constante, sino que en determinado momento ante un aumento del activo se supone que la eficiencia marginal de la inversión cambia su rentabilidad.

Se supone una tasa de interés de mercado. Es decir, hay una tasa a la que en promedio, una unidad superavitaria estaría dispuesta a prestar el volumen promedio de dinero que una unidad deficitaria requiera.

Ajuste sobre la tasa de interés de mercado por variables endógenas al ente. El costo financiero del pasivo incremental fluctúa en relación con el riesgo de insolvencia asumido por la empresa (correlacionado), mientras que el costo del patrimonio inicial se supone constante en el intervalo posible de financiamiento incremental necesario (incorrelacionado), independientemente de este riesgo.

Se supone que el riesgo de insolvencia depende del nivel de endeudamiento asumido por el ente, ceteris paribus todas las demás variables subyacentes e inherentes al negocio (nivel de actividad, 
margen sobre los costos, decisiones de inversión en capital de trabajo y activos fijos, etc.).

Ajuste sobre la tasa de interés de mercado por variables exógenas al ente. El costo financiero del pasivo incremental fluctúa en relación con el volumen dinerario que el inversor estuviera requiriendo al potencial acreedor. De esta manera, a medida que el volumen de financiamiento requerido al acreedor se aleja o acerca más del volumen que en promedio se prestaría a la tasa de mercado, el costo del préstamo aumenta o disminuye, respectivamente.

El costo financiero del pasivo operativo y del patrimonio mínimo requerido no se considera una variable relevante en la determinación del nivel de mezcla óptima. Ambos costos son analizados independientemente de la decisión incremental de financiar la porción no cubierta de la inversión, con fondos de terceros propietarios o no propietarios. Sin embargo, tanto el volumen de pasivos operativos como el volumen de patrimonio mínimo inciden en la determinación de la tasa de interés efectiva del ente, ya que ambos contribuyen a determinar el nivel de endeudamiento resultante.

La tasa de costo de oportunidad de los propietarios no fluctúa en el rango relevante de decisión incremental de la inversión. Esto se supone en razón de la inexistencia de alternativas suficientes y accesibles para los propietarios en caso de que desplazaran la decisión de llevar a cabo la inversión.

\section{Funciones del modelo}

Simbología a utilizar:

$$
\begin{aligned}
C P T_{i}= & \text { Costo financiero total de los fondos } \\
& \text { incrementales }
\end{aligned}
$$

$\mathrm{CP}_{i}=$ Costo financiero de los fondos incrementales provenientes de los propietarios

$P N_{m}=$ Patrimonio mínimo

$P N_{i}=$ Patrimonio incremental

$k=$ Tasa de costo del capital propio

$C T_{i}=$ Costo financiero de los fondos incrementales provenientes de terceros no propietarios

$P_{m}=$ Pasivo mínimo (deudas operativas)

$P_{i} \quad=$ Pasivo incremental

$P_{x}=$ Magnitud dineraria a la que cualquier acreedor -independientemente de las variables endógenas de un ente-prestaría dinero a la tasa $i_{i}$ (tasa de mercado)

$i_{i}=$ Tasa de costo del pasivo financiero incremental (tasa de mercado)

$t \quad=$ Tasa de impuesto a las ganancias

$d_{1}=$ Desviación estándar resultante de la distribución que asumen las tasas de interés para un mismo deudor potencial ante distintos niveles de riesgo de insolvencia asociados a un nivel de endeudamiento

$\beta=$ Coeficiente de correlación entre el riesgo por insolvencia y el nivel de endeudamiento resultante, el cual asume este comportamiento: $0<\mathrm{B}<1$

$d_{2}=$ Coeficiente de ajuste de la tasa de interés por cada unidad monetaria diferencial que el acreedor estuviera dispuesto a prestar al inversor por encima o por debajo del valor que en promedio se prestaría a la tasa de mercado

$A=$ Inversión necesaria

$A_{i}=$ Financiamiento incremental (porción de la inversión necesaria no cubierta 
con deudas operativas ni con capital mínimo aportado por los propietarios)

a. Determinación del costo capital propio (considerando que el costo del $P N_{m}$ no es relevante a los fines del objeto definido en el modelo)

$C P_{i}=P N_{i} * k$

Dado que a los fines del análisis el objeto es validar el costo marginal que genera cada peso adicional de financiamiento por una $\mathrm{u}$ otra fuente alternativa, se torna relevante observar la expresión derivada de esta función $C P$ respecto de $P N_{i}$ (variable independiente) que resulta:

$\frac{\partial C P_{i}}{\partial P N_{i}}=k$

Se observa que el cambio marginal en el costo de las fuentes de financiamiento "fondos de propietarios" es constante e igual a $k$ por cada unidad monetaria adicional que los mismos incorporen a la estructura de financiamiento.

De esta manera, se considera solo el costo de financiamiento incremental, el cual es controlable y relevante para la decisión de financiamiento y queda expresado así:

$C P_{i}=P N_{i} * k$

b. Determinación del costo de capital de terceros (considerando que el costo del $P_{m}$ no es relevante a los fines del objeto definido en el modelo)

$$
C T_{i}=P I\left\{\left[i_{i}+d_{1} * \beta *\left(\frac{P_{i}+P_{m}}{P N_{i}+P N_{m}}\right)+d_{2} *\left(P_{i}-P_{x}\right)\right] *(1-t)\right\}
$$

Se realizan algunas operaciones algebraicas para reescribir la expresión en la forma de una función cuadrática:

$$
\begin{aligned}
C T_{i}= & P_{i} * i_{i} *(1-t)+d_{1} * \beta *(1-t) \\
& *\left(\frac{P_{i}^{2}+P_{i} P_{m}}{P N_{i}+P N_{m}}\right)+d_{2} *(1-t) \\
& *\left(P_{i}^{2}-P_{i} P_{x}\right)
\end{aligned}
$$

Luego, se reordenan los términos de primer orden y los de segundo orden:

$$
\begin{aligned}
C T_{i} & =P_{i} *\left[i_{i}-P_{x} d_{2}+\frac{d_{1} \beta P_{m}}{P N_{i}+P N_{m}}\right] \\
& *(1-t)+P_{i}^{2}\left[\left(\frac{d_{1} \beta}{P N_{i}+P N_{m}}+d_{2}\right)\right] *(1-t)
\end{aligned}
$$

Dado que a los fines del análisis el objeto es validar el costo marginal que genera el financiamiento adicional por una u otra fuente alternativa, se torna relevante la expresión derivada de esta función CT respecto de PI (variable independiente), que resulta:

$$
\begin{aligned}
\frac{\partial C T}{\partial P I}= & {\left[i_{i}-P_{x} d_{2}+\frac{d_{1} \beta P_{m}}{P N_{i}+P N_{m}}\right] } \\
& *(1-t)+2 P_{i}\left[\left(\frac{d_{1} \beta}{P N_{i}+P N_{m}}+d_{2}\right) *(1-t)\right]
\end{aligned}
$$

Se observa que el cambio marginal en el costo de las fuentes de financiamiento "fondos de terceros" no es constante, sino que es una función en relación con el valor que 
asuma el pasivo incremental que adquiera el ente. Se puede observar que este costo tiene una relación:

Positiva y variable respecto de $P_{i}$ Positiva y constante respecto de $i_{i} ; d_{1} ; \beta ; P_{m}$ Negativa y constante respecto de $P_{x} ; t P N_{i}$; $P N_{m}$

De esta manera, se considera solo el costo de financiamiento incremental, el cual es controlable y relevante para la decisión de financiamiento y queda expresado así:

$$
\begin{aligned}
C T_{i} & =P_{i} *\left[i_{i}-P_{x} d_{2}+\frac{d_{1} \beta P_{m}}{P N_{i}+P N_{m}}\right] \\
& *(1-t)+P_{i}^{2}\left[\left(\frac{d_{1} \beta}{P N_{i}+P N_{m}}+d_{2}\right)\right] *(1-t)
\end{aligned}
$$

c. Expresión del patrimonio incremental como función del pasivo incremental $A=(P N M+P N I)+(P M+P I) \rightarrow P I=A-$ $(P N M+P M)-P N I \rightarrow P N I$ $=A-(P N M+P M)-P I$

Se asume que $A, P N_{m}, P_{m}$ son constantes en el modelo y que:

$$
A_{i}=A-P N m-P_{m}
$$

Se define al $P N_{i}$ en relación con el $P_{i}$ como:

$$
P N_{i}=A_{i}-P_{i}
$$

\section{d. Costo}

La función costo total del financiamiento incremental se construye adicionando las ecuaciones obtenidas en $a$ y $b$, para buscar el punto de indiferencia entre ambos costos financieros para un nivel de financiamiento incremental, mediante la minimización de ese costo:

$$
\begin{aligned}
C P T_{i}= & C P_{i}+C T_{i} \\
C P T_{i} & =P_{i}\left[i_{i}-P_{x} d_{2}+\frac{d_{1} \beta P_{m}}{P N_{i}+P N_{m}}\right] *(1-t) \\
& +P_{i}^{2}\left[\left(\frac{d_{1} \beta}{P N_{i}+P N_{m}}+d_{2}\right) *(1-t)\right]+P N_{i} * k
\end{aligned}
$$

$\mathrm{Al}$ sustituir $P N_{i}$ por la expresión obtenida en $c$ :

$$
\begin{aligned}
C P T_{i} & =P_{i}\left[i_{i}-P_{x} d_{2}+\frac{d_{1} \beta P_{m}}{A_{i}-P_{i}+P N_{m}}\right] *(1-t) \\
& +P_{i}^{2}\left[\left(\frac{d_{1} \beta}{A_{i}-P_{i}+P N_{m}}+d_{2}\right) *(1-t)\right]+P N_{i} * k
\end{aligned}
$$

Se busca ahora minimizar este costo total de las fuentes de financiamiento incrementales. A partir de esta función expresada en relación con la variable $P_{i}$ y asignando valores arbitrarios a los parámetros de la función, se puede observar la conveniencia de una de las dos fuentes de financiamiento alternativas ante cada unidad monetaria incremental. De esta manera, el modelo muestra la magnitud marginal de pasivo a tomar, hasta alcanzar un nivel de endeudamiento tal que cada valor monetario adicional de deuda tomado genera una detracción del costo total de financiamiento incremental.

Así, al minimizar este costo, se puede obtener el nivel de endeudamiento que no solo optimiza el costo total de las fuentes controlables de financiación, sino que además será 
el nivel máximo de endeudamiento (ceteris paribus todas las demás variables subyacentes al modelo) que la inversión como tal podría soportar.

\section{Resolución del modelo}

A los fines de validar el modelo desarrollado, se atribuyen arbitrariamente valores a los parámetros definidos en las relaciones

\begin{tabular}{cccc}
\hline \multicolumn{2}{c}{ Parámetros } & \multicolumn{2}{c}{ Parámetros } \\
\hline $\mathrm{A}$ & $100.000,00$ & $\mathrm{i}_{\mathrm{i}}$ & 0,1750000 \\
$\mathrm{~A}_{\mathrm{i}}$ & $70.000,00$ & $\mathrm{t}$ & 0,3500000 \\
$\mathrm{PN}_{\mathrm{m}}$ & $20.000,00$ & $\mathrm{~d}_{1}$ & 0,0979873 \\
$\mathrm{P}_{\mathrm{m}}$ & $10.000,00$ & $\mathrm{~b}$ & 0,9687460 \\
$\mathrm{k}$ & 0,2000000 & $\mathrm{~d}_{2}$ & 0,0000020 \\
\hline
\end{tabular}

A continuación, la tabla resolutiva del modelo y el gráfico muestran el comportamiento de las tasas de costo ( $k$; i y tasa de costo total s/ $\left.A_{i}\right)$. Luego se adiciona una matriz de resumen de distintos escenarios, que denota el nivel óptimo de endeudamiento que tolerarían distintas estructuras de inversión con la tasa de costo total mínimo vinculada. Finalmente, se incorpora el ROA para evaluar conjuntamente el comportamiento de la tasa de costo total de las fuentes de financiamiento incrementales y el desempeño económico del negocio.

A continuación, la tabla 1 exterioriza la solución obtenida al resolver el modelo al asignarle parámetros arbitrarios.

En el ejemplo que antecede, frente a la decisión de invertir $\$ 70.000$, se busca obtener la mezcla de financiamiento que minimice el costo total de la estructura de capital, teniendo en cuenta que a mayor pasivo, este costo aumenta por dos vías: una exógena (porque los acreedores elevan la tasa por el incremento del riesgo) y otra endógena (por el mayor riesgo de insolvencia que asume la empresa). Todo ello, teniendo en cuenta que se requiere un patrimonio mínimo de $\$ 20.000$ y un pasivo que no podrá estar por debajo de $\$ 10.000$ para atender las necesidades operativas. De esta manera, a partir de la resolución de la tabla 1, se llega a la conclusión de que la alternativa más económica para financiar la inversión incremental es obtener $\$ 27.493,81$ por medio de pasivos financieros y el resto $(\$ 42.506,19)$ de capital propio, porque el costo promedio ponderado de 0,168941670 es el menor de la serie, con lo cual la ratio de endeudamiento que se asume para esta decisión es $60 \%$, debido a que el pasivo será 37.493,81 $(10.000+27.493,81)$ y el patrimonio 62.506,19 $(20.000+42.506,19)$. Posteriormente, habrá que verificar que la tasa obtenida esté por debajo de la rentabilidad de la inversión incremental $\left(R O A_{i}\right)$, para que se pueda lograr un valor económico agregado (EVA) positivo.

En el gráfico 1, se puede observar la evolución de las variables de costo del financiamiento, según los valores obtenidos en la tabla 1. De esta manera, para distintos niveles de endeudamiento, se verifica que el costo del capital propio permanece constante, mientras que el costo del pasivo parte de niveles bajos, pero se incrementa significativamente, a medida que el endeudamiento va generando un riesgo mayor. 


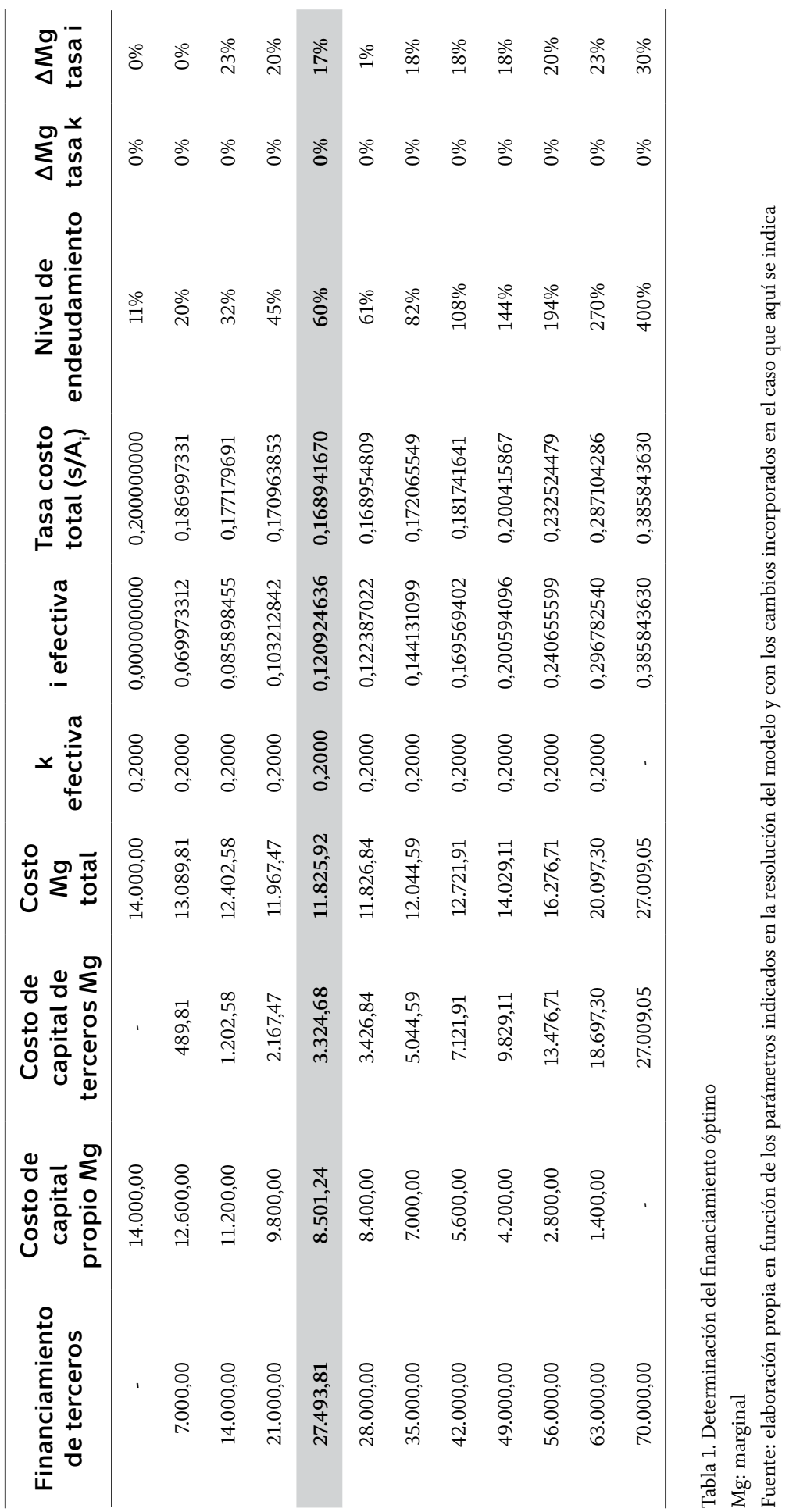




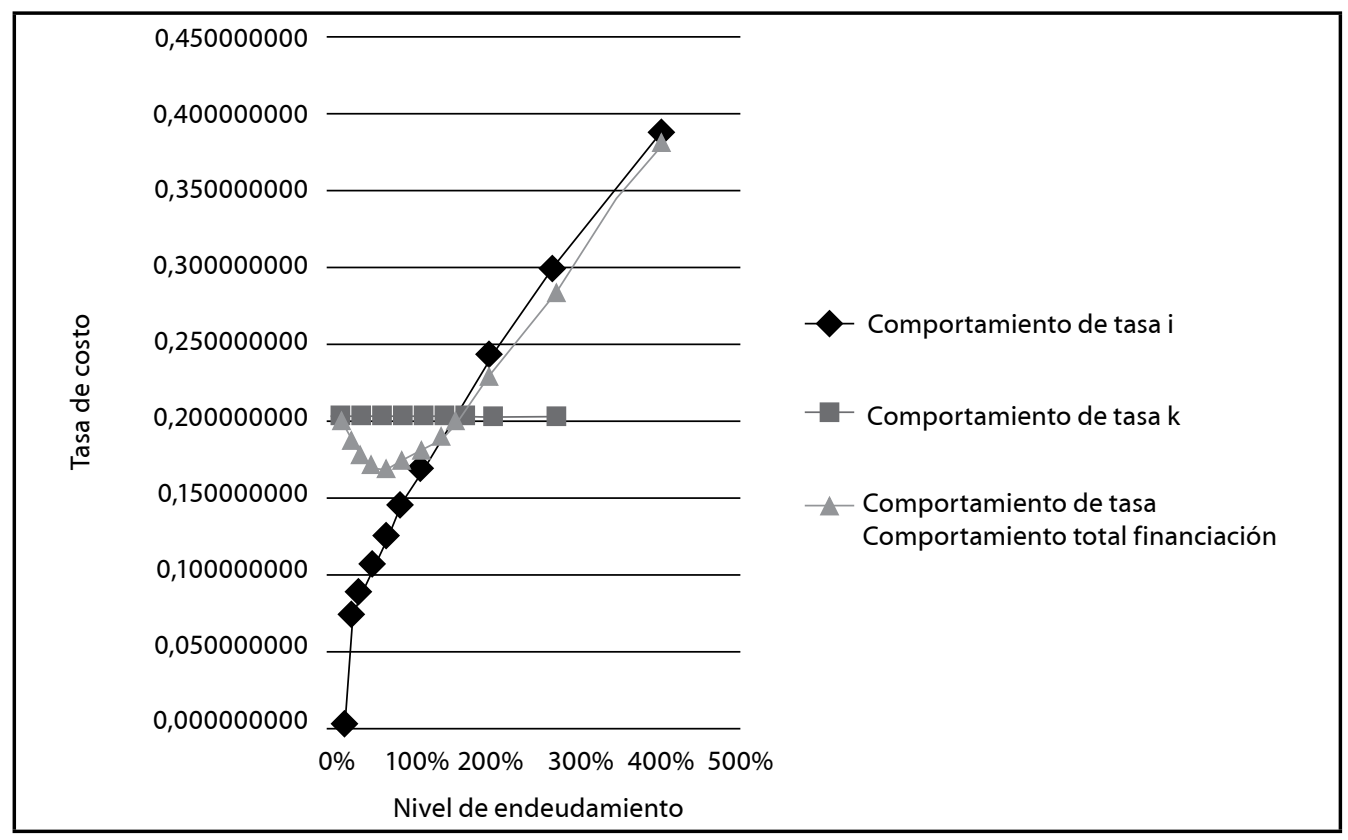

Gráfico 1. Análisis del comportamiento de las tasas de costo de financiamiento Fuente: elaboración propia

En la tabla 2 se realiza una simulación con distintas alternativas de patrimonio y pasivo mínimos para cada tramo de inversión, para mostrar cuál es el nivel de endeudamiento que minimiza el costo del capital.

Así, puede observarse que del último renglón de la segunda columna de restricciones está tomado el ejemplo que se consideró en la resolución del modelo referido a una inversión incremental de $\$ 70.000$, lo que hace un activo total de $\$ 100.000$, habiendo partido de un mínimo de $\$ 30.000$ financiado por el patrimonio y pasivo mínimos.

\begin{tabular}{|c|c|c|c|c|c|}
\hline \multirow[b]{2}{*}{$\begin{array}{l}\text { Inversión } \\
\text { necesaria }\end{array}$} & \multirow[b]{2}{*}{$\begin{array}{l}\text { Variables de } \\
\text { análisis }\end{array}$} & \multicolumn{4}{|c|}{ Restricciones a la mezcla óptima de endeudamiento } \\
\hline & & $\begin{array}{c}P N_{m}=0 \% A \\
P_{m}=0 \% A\end{array}$ & $\begin{array}{c}P N_{m}=20 \% A \\
P_{m}=10 \% A\end{array}$ & $\begin{array}{c}P N_{m}=25 \% A \\
P_{m}=15 \% A\end{array}$ & $\begin{array}{l}P N_{m}=0 \% A \\
P_{m}=45 \% A\end{array}$ \\
\hline \multirow{2}{*}{$10.000,00$} & $\begin{array}{l}\text { Nivel de } \\
\text { endeudamiento }(P / P N)\end{array}$ & $81 \%$ & $91 \%$ & $97 \%$ & $149 \%$ \\
\hline & $\begin{array}{l}\text { Tasa costo total mínimo } \\
\text { (sobre el } A_{i} \text { ) }\end{array}$ & 0,15706 & 0,15122 & 0,15010 & 0,18438 \\
\hline \multirow{2}{*}{$20.000,00$} & $\begin{array}{l}\text { Nivel de } \\
\text { endeudamiento }(P / P N)\end{array}$ & $76 \%$ & $87 \%$ & $93 \%$ & $146 \%$ \\
\hline & $\begin{array}{l}\text { Tasa costo total mínimo } \\
\left(\text { sobre el } A_{i}\right)\end{array}$ & 0,15956 & 0,15378 & 0,15257 & 0,18488 \\
\hline
\end{tabular}




\begin{tabular}{|c|c|c|c|c|c|}
\hline \multirow[b]{2}{*}{$\begin{array}{l}\text { Inversión } \\
\text { necesaria }\end{array}$} & \multirow[b]{2}{*}{$\begin{array}{c}\text { Variables de } \\
\text { análisis }\end{array}$} & \multicolumn{4}{|c|}{ Restricciones a la mezcla óptima de endeudamiento } \\
\hline & & $\begin{array}{c}P N_{m}=0 \% A \\
P_{m}=0 \% A\end{array}$ & $\begin{array}{c}P N_{m}=20 \% A \\
P_{m}=10 \% A\end{array}$ & $\begin{array}{c}P N_{m}=25 \% A \\
P_{m}=15 \% A\end{array}$ & $\begin{array}{l}P N_{m}=0 \% A \\
P_{m}=45 \% A\end{array}$ \\
\hline \multirow{2}{*}{$25.000,00$} & $\begin{array}{l}\text { Nivel de } \\
\text { endeudamiento }(P / P N)\end{array}$ & $74 \%$ & $85 \%$ & $91 \%$ & $145 \%$ \\
\hline & $\begin{array}{l}\text { Tasa costo total mínimo } \\
\text { (sobre el } A_{i} \text { ) }\end{array}$ & 0,16075 & 0,15499 & 0,15375 & 0,18513 \\
\hline \multirow{2}{*}{$30.000,00$} & $\begin{array}{l}\text { Nivel de } \\
\text { endeudamiento }(P / P N)\end{array}$ & $71 \%$ & $83 \%$ & $90 \%$ & $144 \%$ \\
\hline & $\begin{array}{l}\text { Tasa costo total mínimo } \\
\text { (sobre el } A_{i} \text { ) }\end{array}$ & 0,16189 & 0,15617 & 0,15489 & 0,18536 \\
\hline \multirow{2}{*}{$40.000,00$} & $\begin{array}{l}\text { Nivel de } \\
\text { endeudamiento }(P / P N)\end{array}$ & $67 \%$ & $79 \%$ & $86 \%$ & $142 \%$ \\
\hline & $\begin{array}{l}\text { Tasa costo total mínimo } \\
\text { (sobre el } A_{i} \text { ) }\end{array}$ & 0,16406 & 0,15841 & 0,15706 & 0,18582 \\
\hline \multirow{2}{*}{$50.000,00$} & $\begin{array}{l}\text { Nivel de } \\
\text { endeudamiento }(P / P N)\end{array}$ & $63 \%$ & $75 \%$ & $82 \%$ & $140 \%$ \\
\hline & $\begin{array}{l}\text { Tasa costo total mínimo } \\
\text { (sobre el } A_{i} \text { ) }\end{array}$ & 0,16606 & 0,16050 & 0,15910 & 0,18625 \\
\hline \multirow{2}{*}{$60.000,00$} & $\begin{array}{l}\text { Nivel de } \\
\text { endeudamiento }(P / P N)\end{array}$ & $59 \%$ & $72 \%$ & $79 \%$ & $118 \%$ \\
\hline & $\begin{array}{l}\text { Tasa costo total mínimo } \\
\text { (sobre el } A_{i} \text { ) }\end{array}$ & 0,16792 & 0,16244 & 0,16100 & 0,18806 \\
\hline \multirow{2}{*}{$70.000,00$} & $\begin{array}{l}\text { Nivel de } \\
\text { endeudamiento }(P / P N)\end{array}$ & $55 \%$ & $69 \%$ & $76 \%$ & $137 \%$ \\
\hline & $\begin{array}{l}\text { Tasa costo total mínimo } \\
\text { (sobre el } A_{i} \text { ) }\end{array}$ & 0,16963 & 0,16425 & 0,16277 & 0,18706 \\
\hline \multirow{2}{*}{$75.000,00$} & $\begin{array}{l}\text { Nivel de } \\
\text { endeudamiento (P/PN) }\end{array}$ & $54 \%$ & $67 \%$ & $74 \%$ & $136 \%$ \\
\hline & $\begin{array}{l}\text { Tasa costo total mínimo } \\
\text { (sobre el } A_{i} \text { ) }\end{array}$ & 0,17043 & 0,16510 & 0,16361 & 0,18725 \\
\hline \multirow{2}{*}{$80.000,00$} & $\begin{array}{l}\text { Nivel de } \\
\text { endeudamiento }(P / P N)\end{array}$ & $52 \%$ & $65 \%$ & $73 \%$ & $135 \%$ \\
\hline & $\begin{array}{l}\text { Tasa costo total mínimo } \\
\text { (sobre el } A_{i} \text { ) }\end{array}$ & 0,17121 & 0,16593 & 0,16443 & 0,18743 \\
\hline \multirow{2}{*}{$90.000,00$} & $\begin{array}{l}\text { Nivel de } \\
\text { endeudamiento }(P / P N)\end{array}$ & $49 \%$ & $63 \%$ & $70 \%$ & $134 \%$ \\
\hline & $\begin{array}{l}\text { Tasa costo total mínimo } \\
\text { (sobre el } A_{i} \text { ) }\end{array}$ & 0,17266 & 0,16749 & 0,16597 & 0,18779 \\
\hline \multirow{2}{*}{$100.000,00$} & $\begin{array}{l}\text { Nivel de } \\
\text { endeudamiento }(P / P N)\end{array}$ & $46 \%$ & $60 \%$ & $68 \%$ & $132 \%$ \\
\hline & $\begin{array}{l}\text { Tasa costo total mínimo } \\
\left(\text { sobre el } A_{i}\right)\end{array}$ & 0,17401 & 0,16894 & 0,16741 & 0,18813 \\
\hline
\end{tabular}

Tabla 2. Nivel de endeudamiento para cada tramo de inversión

Fuente: elaboración propia en función de los parámetros indicados en la resolución del modelo y con los cambios incorporados en el caso que se indica aquí 
A continuación, para cada tramo de inversión, la tabla 3 indica cuál es el costo mínimo (para los parámetros $P N_{m}=20 \% A$ y $P_{m}=10 \% A$ ) respecto a un valor supuesto del $R_{0} A_{i}$ (rentabilidad de la inversión marginal).

\begin{tabular}{cccc}
\hline $\begin{array}{c}\text { Inversión } \\
\text { total }\end{array}$ & $\begin{array}{c}\text { Tasa de costo total mínimo para cada } \\
\text { nivel óptimo de endeudamiento, } \\
\text { sujeto a la restricción: } \\
\boldsymbol{P N}_{m}=\mathbf{2 0 \% A}\end{array}$ & ROA & $\begin{array}{c}\text { Diferencia entre el } \\
\boldsymbol{P}_{m}=10 \% \boldsymbol{A} \text { y el costo total } \\
\text { mínimo }\end{array}$ \\
\hline $10.000,00$ & 0,1512164 & 0,2210699 & 0,0698535 \\
$20.000,00$ & 0,1537766 & 0,2263272 & 0,0725505 \\
$30.000,00$ & 0,1561722 & 0,2535270 & 0,0973548 \\
$40.000,00$ & 0,1584094 & 0,2882818 & 0,1298724 \\
$50.000,00$ & 0,1604954 & 0,3372849 & 0,1767895 \\
$60.000,00$ & 0,1624381 & 0,3136503 & 0,1512121 \\
$70.000,00$ & 0,1642456 & 0,2131520 & 0,0489064 \\
$80.000,00$ & 0,1659264 & 0,1482269 & $-0,0176996$ \\
$90.000,00$ & 0,1674890 & 0,1176767 & $-0,0498123$ \\
$100.000,00$ & 0,1689417 & 0,0800000 & $-0,0889417$ \\
\hline
\end{tabular}

Tabla 3. Diferencia entre el ROA y el costo total mínimo

Fuente: elaboración propia en función de los parámetros indicados en la resolución del modelo y con los cambios incorporados en el caso que se indica aquí

En función del comportamiento que se observa en la tabla 3, el punto donde la diferencia entre el ROA marginal y el costo total mínimo se hace máxima, se produce para una inversión de $\$ 50.000$, que relacionada con la tabla 2 indica que el nivel de endeudamiento para ese tramo es del $75 \%$.

Como para $\$ 50.000$, el pasivo mínimo deberá ser de $\$ 5.000$ y el patrimonio de partida $\$ 10.000$, arroja que la inversión incremental que maximiza la diferencia es $\$ 35.000$.

Siendo que el activo total es $\$ 50.000$, podemos igualarlo al patrimonio, y pasivo escribiendo este último en función del anterior:

$$
50.000=0,75 \mathrm{PN}+\mathrm{PN}
$$

Resolviendo, queda:

$$
\mathrm{PN}=50.000 / 1,75=28.571
$$

Siendo el pasivo: $50.000-28.571=21.429$

Con lo cual, la inversión total debería financiarse $\$ 21.429$ con pasivo ( $\$ 5.000$ operativos y el resto, financieros) y $\$ 28.571$ con recursos propios ( $\$ 10.000$ iniciales y el resto con aportes o resultados no distribuidos).

Si se decide llevar el activo a $\$ 80.000$, el rendimiento de los fondos incrementales estaría por debajo de su costo marginal.

A continuación, se grafica la situación planteada en la tabla 3 y se puede observar claramente el momento en que la brecha entre el ROA y el costo del capital se amplía positivamente y el punto donde la situación se invierte. 


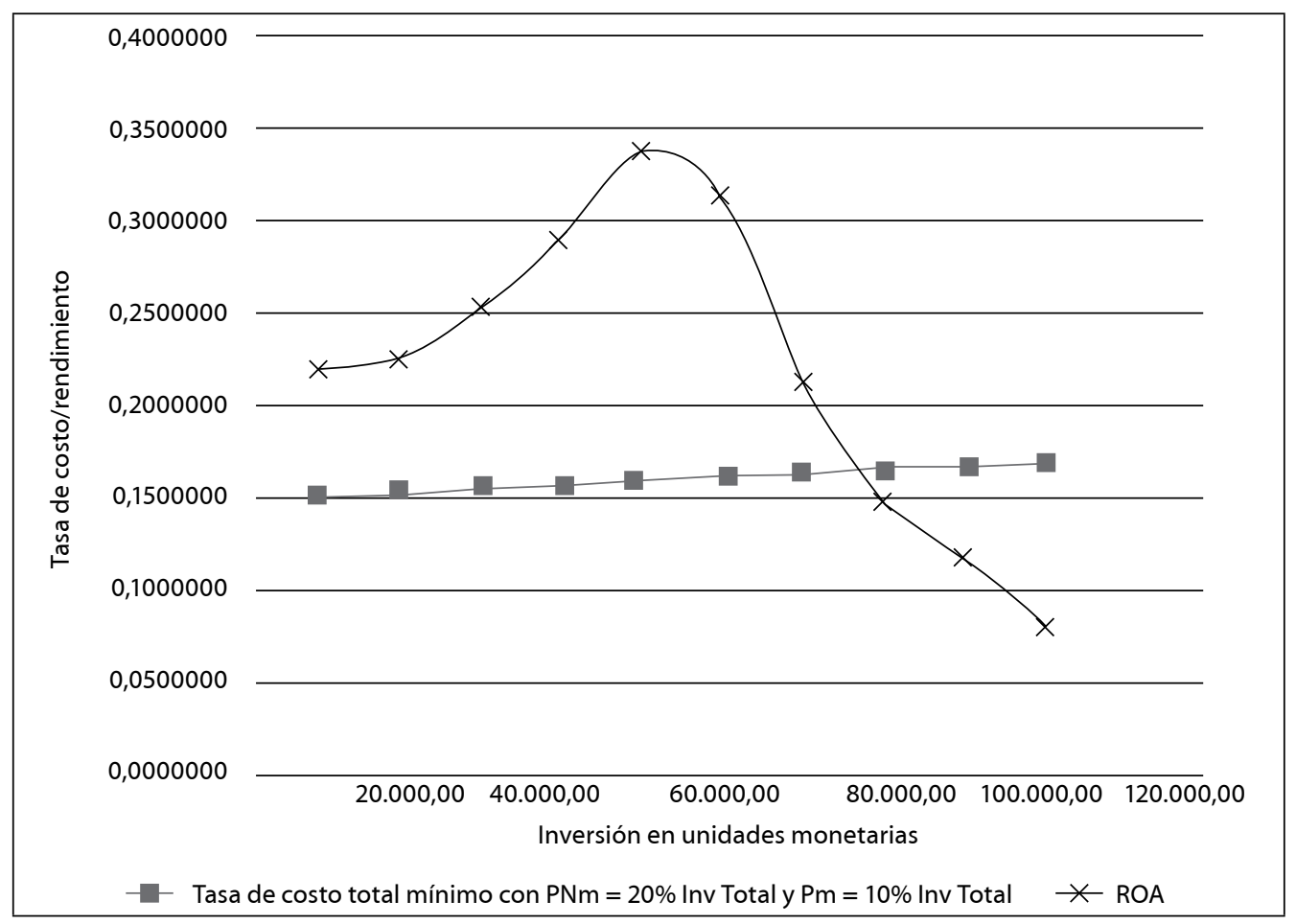

Gráfico 2. Diferencia entre el ROA y el costo total mínimo Fuente: elaboración propia

\section{Conclusiones}

En países en vías de desarrollo y en particular en Latinoamérica, el financiamiento es una decisión tan importante como la inversión, y en algunos casos más aún que esta última. Aunado a lo anterior, las dificultades que tienen las pyme al momento de requerir los fondos para sus actividades, hacen necesario tener claridad sobre las variables que incidirán en su desempeño futuro, para que estas entidades no queden totalmente expuestas a quebrantos y situaciones de insolvencia.

Si bien en algunos casos puede haber políticas públicas de fomento a las pequeñas y medianas empresas, para que obtengan préstamos a tasas subsidiadas, en muchos casos se observa que las entidades financieras ejercen un racionamiento del crédito hacia las pyme. Por otra parte, no es común que estas tengan acceso al mercado de capitales. Si a los hechos mencionados se les agrega que en diversas circunstancias los contextos económicos de algunos países latinoamericanos no son estables, resolver el problema de la estructura de capital constituye un verdadero desafío para el administrador financiero de una pyme.

Hay que considerar que cuestiones intrínsecas a este tipo de organizaciones, como la renuencia a compartir el control con otros socios o accionistas y el riesgo a perder la empresa por elevado endeudamiento, le agregan aspectos diferenciales al momento de tomar la 
decisión sobre la mezcla de las fuentes de financiamiento.

Por ello, se percibe la necesidad de generar un modelo matemático que, partiendo de determinadas premisas inherentes a la situación planteada en este trabajo, pueda establecer ante cada tramo de inversión incremental el menor costo de financiamiento, de manera que este pueda ser comparado con la rentabilidad del activo y observar el valor económico agregado que se produce al tomar la decisión.

En este caso, el modelo desarrollado se apoya en las siguientes premisas: capital propio mínimo, por debajo del cual los acreedores no estarán dispuestos a financiar; pasivo mínimo, asociado al financiamiento de las actividades operativas; el costo del financiamiento de terceros no es constante sino que crece por dos vías; a mayor desembolso, los acreedores suben la tasa por el riesgo e internamente, la organización considera el peligro que implicaría caer en una situación de insolvencia.

El riesgo endógeno es una cuestión importante, que en el modelo se considera un parámetro en función de los niveles de endeudamiento. La potencial desaparición de una pyme es una cuestión más sensible para sus integrantes que la de las grandes empresas, donde puede haber una disociación entre la propiedad y la gestión.

Así como cada empresa debe buscar la mejor estructura financiera para su situación particular, esta va a diferir de acuerdo al intervalo de inversión que se esté analizando, ya que en las pyme los costos pueden variar significativamente por cada tramo, hecho este que no se produce habitualmente en las corporaciones que trabajan en mercados desarrollados.
Se espera que la herramienta desarrollada en este trabajo contribuya a mejorar la gestión financiera de las pyme. A partir de aquí, podrían realizarse otros estudios que incluyan las distintas variables de riesgo (operativo y financiero) mencionadas en el trabajo y que, incorporadas al modelo matemático, permitirían conseguir mayor precisión en la determinación de la estructura de capital.

\section{Referencias}

Akerlof, George A. \& Shiller, Robert J. (2009). Animal Spirits: Cómo influye la psicología humana en la economía. Barcelona: Gestión 2000.

Bebczuk, Ricardo (2010). Aplicaciones a las finanzas corporativas. En Ricardo Bebczuk (ed.). Progresos en finanzas, 11-26. Buenos Aires: Asociación Argentina de Economía Política, AAEP. Disponible en: http:// www.aaep.org.ar/publicaciones/download/ progresos_finanzas.pdf

Bodie, Zvi; Merton, Robert C. \& Vinitzky, Guillermo M. (2006). Finanzas y gestión. México: Pearson.

Brealey, Richard A.; Myers, Stewart C. \& Allen, Franklin (2006). Principios de finanzas corporativas. Madrid: McGraw-Hill.

Brennan, Michael J. \& Schwartz, Eduardo S.

(1978). Corporate Income Taxes, Valuation, and the Problem of Optimal Capital Structure. Journal of Business, 51 (1), 103-114. Disponible en: http://www.anderson.ucla.edu/ faculty/eduardo.schwartz/articles/7.pdf Brighi, Paola \& Torluccio, Giuseppe (2007). Evidence on Funding Decisions by Italian SMEs. A Self-Selection Model? Working 
paper University of Bologna, Department of Management and CREDIF. Disponible en: papers.ssrn.com/sol3/papers.cfm?abstract_ id $=1629988$

Briozzo, Anahí \& Vigier, Hernán (2008). A Demand-Side Approach to SME's Capital Structure. Evidence from Argentina. Midwest Finance Association $57^{\text {th }}$ Annual Meeting San Antonio (Texas), February 27-March 31, 2008.

Briozzo, Anahí \& Vigier, Hernán (2009). La estructura de capital de las pequeñas y medianas empresas argentinas. Estudios Económicos, 26 (53), 1-55.

Dapena, José Pablo \& Dapena, Juan Lucas (2003). Sistemas de información en PYMES y acceso al crédito en contextos de asimetría de información. Serie Documentos de Trabajo. Área: Negocios y Finanzas, 252. Buenos Aires: Universidad del Centro de Estudios Macroeconómicos de Argentina, UCEMA. Disponible en: http://cefim.com.ar/Informacion_Asimetrica.pdf, http://www.ucema. edu.ar/publicaciones/doc_trabajo.php/252

Diamond, Douglas W. (1989). Reputation Acquisition in Debt Markets. Journal of Political Economy, 97 (4), 828-862. Disponible en: http://core.ac.uk/download/ pdf/6519112.pdf

Drimer, Roberto (2011). Teoría del financiamiento. Buenos Aires: Buyatti.

Durand, David (1952). Cost of Debt and Equity Funds for Business: Trends and Problems of Measurement. A chapter in Conference on Research in Business Finance, 215-262. New York: National Bureau of Economic Research. Disponible en: http://www.nber. org/chapters/c4790.pdf
Fama, Eugene \& Miller, Merton H. (1972). The Theory of Finance. New York: Holt, Rinehart and Winston.

Fanelli, José María; Bebczuk, Ricardo N. \& Pradelli, Juan J. (2002). Determinants and Consequences of Financial Constraints Facing Firms in Argentina. Inter-American Development Bank, Latin American Research Network (Banco Interamericano de Desarrollo, Red de Centros de Investigación), Research Network Working paper R-453. Disponible en: http://www.iadb.org/res/publications/ pubfiles/pubR-453.pdf

Forte, Denis; Barros, Lucas Ayres B. de C. \& Nakamura, Wilson Toshiro (2013). Determinants of the capital structure of small and medium sized Brazilian enterprises. BAR - Brazilian Administration Review, 10 (3), 347-369. Disponible en: http://papers.ssrn.com/sol3/papers. cfm?abstract_id=2304678

Fundación de Investigaciones Económicas Latinoamericanas, FIEL (1996). Las pequeñas y medianas empresas en la Argentina. Buenos Aires: Fundación de Investigaciones Económicas Latinoamericanas, FIEL. Disponible en: http://www.fiel.org/publicaciones/Libros/pyme.pdf

Jensen, Michael (1986). Agency Costs of Free Cash Flow, Corporate Finance, and Takeovers. American Economic Review, 76 (2), 323-329. Disponible en: http://papers.ssrn. com/sol3/papers.cfm?abstract_id=99580 Jensen, Michael \& Meckling, William H. (1976). Theory of the Firm: Managerial Behavior, Agency Cost and Ownership Structure. Journal of Financial Economics, 3 (4), 
305-360. Disponible en: http://papers.ssrn. com/sol3/papers.cfm?abstract_id=94043

Kim, E. Han (1978). A Mean-Variance Theory of Optimal Capital Structure and Corporate Debt Capacity. The Journal of Finance, 33 (1), 45-63. Disponible en: http://webuser.bus. umich.edu/ehkim/articles/1978-03_MeanVariance_JF.pdf

Kraus, Alan \& Litzenberger, Robert H. (1973). A State-Preference Model of Optimal Financial Leverage. The Journal of Finance, 28 (4), 911-922.

Leach, Peter (2010). La empresa familiar. Buenos Aires: Granica.

LeRoy, Stephen F. (2004). Rational Exuberance. Journal of Economic Literature, 42 (3), 783-804. Disponible en: http://econ.ucsb. edu/ sleroy/downloads/surapr14.pdf

López-Dumrauf, Guillermo (2003). Finanzas corporativas. Buenos Aires: Grupo Guía.

Mac an Bhaird, Ciarán \& Lucey, Brian (2011). An Empirical Investigation of the Financial Growth Lifecycle. Journal of Small Business and Enterprise Development, 18 (4), 715-731. Disponible en: http://mpra.ub.uni-muenchen.de/61948/

Miller, Merton H. (1977). Debt and Taxes. The Journal of Finance, 32 (2), 261-275. Disponible en: https://www2.bc.edu/thomas-chemmanur/phdfincorp/MF891\%20papers/ Miller\%20tax\%201977.pdf, https://www2. bc.edu/thomas-chemmanur/phdfincorp/ MF891\%20papers/Miller\%20tax\%201977.pdf Modigliani, Franco \& Miller, Merton H. (1958). The Cost of Capital, Corporation Finance and the Theory of Investment. American Economic Review, 48
(3), 261-297. Disponible en: https:// www2.bc.edu/ chemmanu/phdfincorp/ MF891\%20papers/MM1958.pdf

Modigliani, Franco \& Miller, Merton H. (1959). The Cost of Capital, Corporation Finance and the Theory of Investment: Reply. American Economic Review, 49 (4), 655-669. Disponible en: http://webkuliah.unimedia. ac.id/ebook/files/modigliani-miller2.pdf Modigliani, Franco \& Miller, Merton H. (1963). Corporate Income Taxes and the Cost of Capital: A Correction. American Economic Review, 53 (3), 433-443. Disponible en: https://www2.bc.edu/ chemmanu/phdfincorp/MF891\%20papers/MM1963.pdf

Myers, Stewart C. (1984). The Capital Structure Puzzle. The Journal of Finance, 39 (3), 575-592. Disponible en: http://dspace. mit.edu/bitstream/handle/1721.1/2078/ SWP-1548-15376697.pdf, http://www.nber. org/papers/w1393

Myers, Stewart C. \& Majluf, Nicholas S. (1984). Corporate Financing and Investment Decisions when Firms Have Information the Investors Do Not Have. National Bureau of Economic Research, NBER. Working Paper 1396. Disponible en: http://citeseerx.ist.psu. edu/viewdoc/download?doi=10.1.1.334.715 $4 \&$ rep $=$ rep $1 \&$ type $=$ pdf

Palepu, Krishna G.; Healy, Paul M. \& Bernard, Victor L. (2002). Análisis y valuación de negocios: mediante estados financieros. México: Thomson.

Pascale, Ricardo (2009). Decisiones financieras. Buenos Aires: Pearson.

Pérez, Jorge Orlando (2013). El análisis de estados financieros bajo distintas perspectivas. 
Ponencia realizada en la XXX Conferencia Interamericana de Contabilidad, 1-3 de diciembre de 2013, Punta del Este, Uruguay. Publicado por la Asociación Interamericana de Contabilidad. Disponible en: http:// www.cpcecba.org.ar/media/img/paginas/ EL\%20AN\%C3\%81LISIS\%20DE\%20 ESTADOS\%20FINANCIEROS\%20BAJO\%20 DISTINTAS\%20PERSPECTIVAS.pdf

Petersen, Mitchell \& Rajan, Raghuram G. (1994). The Benefits of Lending Relationships: Evidence from Small Business Data. The Journal of Finance, 49 (1), 3-37. Disponible en: https://www2. bc.edu/ strahan/petersen\%20rajan\%20 1994.pdf

Romano, Claudio; Tanewski, George A. \& Smyrnios, Kosmas (2000). Capital Structure Decision Making: A Model for Family Business. Journal of Business Venturing, 16, 285-310. Disponible en: http://down.cenet. org.cn/upfile/35/200767132512170.pdf

Schweitzer, Maurice (2008). 'Feeling the Love' (or Anger): How Emotions Can Distort the Way We Respond to Advice. Universia Knowledge Wharton (boletín electrónico). Disponible en: http://wharton.universia. net/index.cfm?fa =viewArticle\&id $=1592$ \&language $=$ english\&specialid $=$, http:// knowledge.wharton.upenn.edu/article/ feeling-the-love-or-anger-how-emotionscan-distort-the-way-we-respond-to-advice/

Stein, Edward (1997). Can We Be Justified in Believing that Humans Are Irrational? Philosophy and Phenomenological Research, 57 (3), 545-565. Disponible en: http://papers.ssrn. com/sol3/papers.cfm?abstract_id=1930582
Stiglitz, Joseph \& Weiss, Andrew (1981). Credit Rationing in Markets with Imperfect Information. American Economic Review, 71 (3), 393-410. Disponible en: http://pascal.iseg. utl.pt/ aafonso/eif/pdf/crrinf81.pdf

Titman, Sheridan \& Wessels, Roberto (1988). The Determinants of Capital Structure. The Journal of Finance, 43 (1), 1-19. Disponible en: http://epge.fgv.br/we/MD/FinancasCorp orativas $/ 2006$ ?action $=$ AttachFile $\&$ do $=$ get $\&$ target $=$ titmanwessels.pdf

Zoppa, Adrian \& McMahon, Richard G. P. (2002). Pecking Order Theory and the Financial Structure of Manufacturing SME from Australia's Business Longitudinal Survey. The Flinders University of South Australia, School of Commerce, Research Paper Series 02-1. Disponible en: http://www. flinders.edu.au/sabs/business-files/research/ papers/2002/02-01.pdf

- Fecha de recepción: 15 de septiembre de 2014

- Fecha de aceptación: 26 de marzo de 2015

- Disponible en línea: 30 de abril de 2015

\section{Para citar este artículo}

Pérez, Jorge Orlando; Bertoni, Martín; Sattler, Silvana Andrea \& Terreno, Dante Domingo (2015). Bases para un modelo de estructura de financiamiento en las pyme latinoamericanas. Cuadernos de Contabilidad, 16 (40), 179-204. http://dx.doi. org/10.11144/Javeriana.cc16-40.bpme 